\title{
Video Article \\ Optimization of High Grade Glioma Cell Culture from Surgical Specimens for Use in Clinically Relevant Animal Models and 3D Immunochemistry
}

\author{
Laura A. Hasselbach ${ }^{1}$, Susan M. Irtenkauf ${ }^{1}$, Nancy W. Lemke ${ }^{1}$, Kevin K. Nelson ${ }^{1}$, Artem D. Berezovsky ${ }^{1}$, Enoch T. Carlton ${ }^{1}$, Andrea D. Transou ${ }^{1}$, \\ Tom Mikkelsen ${ }^{1}$, Ana C. deCarvalho ${ }^{1}$ \\ ${ }^{1}$ Department of Neurosurgery, Henry Ford Hospital
}

Correspondence to: Ana C. deCarvalho at Adecarv1@hfhs.org

URL: https://www.jove.com/video/51088

DOI: doi: $10.3791 / 51088$

Keywords: Medicine, Issue 83, Primary Cell Culture, animal models, Nervous System Diseases, Neoplasms, glioblastoma, neurosphere, surgical specimens, long-term self-renewal

Date Published: $1 / 7 / 2014$

Citation: Hasselbach, L.A., Irtenkauf, S.M., Lemke, N.W., Nelson, K.K., Berezovsky, A.D., Carlton, E.T., Transou, A.D., Mikkelsen, T., deCarvalho, A.C. Optimization of High Grade Glioma Cell Culture from Surgical Specimens for Use in Clinically Relevant Animal Models and 3D Immunochemistry. J. Vis. Exp. (83), e51088, doi:10.3791/51088 (2014).

\section{Abstract}

Glioblastomas, the most common and aggressive form of astrocytoma, are refractory to therapy, and molecularly heterogeneous. The ability to establish cell cultures that preserve the genomic profile of the parental tumors, for use in patient specific in vitro and in vivo models, has the potential to revolutionize the preclinical development of new treatments for glioblastoma tailored to the molecular characteristics of each tumor.

Starting with fresh high grade astrocytoma tumors dissociated into single cells, we use the neurosphere assay as an enrichment method for cells presenting cancer stem cell phenotype, including expression of neural stem cell markers, long term self-renewal in vitro, and the ability to form orthotopic xenograft tumors. This method has been previously proposed, and is now in use by several investigators. Based on our experience of dissociating and culturing 125 glioblastoma specimens, we arrived at the detailed protocol we present here, suitable for routine neurosphere culturing of high grade astrocytomas and large scale expansion of tumorigenic cells for preclinical studies. We report on the efficiency of successful long term cultures using this protocol and suggest affordable alternatives for culturing dissociated glioblastoma cells that fail to grow as neurospheres. We also describe in detail a protocol for preserving the neurospheres 3D architecture for immunohistochemistry. Cell cultures enriched in CSCs, capable of generating orthotopic xenograft models that preserve the molecular signatures and heterogeneity of GBMs, are becoming increasingly popular for the study of the biology of GBMs and for the improved design of preclinical testing of potential therapies.

\section{Video Link}

The video component of this article can be found at https://www.jove.com/video/51088/

\section{Introduction}

Glioblastoma (GBM), a WHO grade IV astrocytoma, is the most prevalent and aggressive primary brain tumor. Diverse developmental phenotypes are adopted by GBM tumor cells, including cells exhibiting "cancer stem cell" (CSC) characteristics, such as the expression of neural stem cell (NSC) markers, long term self-renewal, and the potential to give rise to more differentiated cells expressing astrocytic markers and forming the bulk of the tumor ${ }^{-3}$. While clarification is still needed regarding CSC molecular identity and clinical implications, the focus of the present work is on the operational definition of CSC: long term self-renewal in vitro and the ability to differentiate and reproduce the original tumor upon orthotopic implantation in immunocompromised rodents.

Glioblastoma cells have been cultured for decades in traditional medium containing $10 \%$ fetal bovine serum (FBS) and several high passage commercially available serum cultured GBM cell lines are tumorigenic in immunocompromised rodents, but there is considerable genomic and molecular divergence from the original tumor ${ }^{4}$, limiting their use as clinically relevant models. More recently, cells with stem/progenitor phenotype responsive to EGF and bFGF were identified in $\mathrm{GBMs}^{2}$. Subsequently, dissociated GBM tumor samples were cultured in a serum-free medium $^{3}$, originally formulated for the selection and expansion of neural stem cells from the adult mammalian brain ${ }^{5}$. These culture conditions hinder the growth of most nonneoplastic and more differentiated tumor cell populations, while favoring the growth of stem and progenitor cells as floating multicellular spheroids, or neurospheres ${ }^{3}$, mimicking the behavior of adult mammalian neural stem cells ${ }^{5}$. Comprehensive side by side comparison of primary GBM cells cultured in either neurosphere medium supplemented with growth factors (NMGF) or in the traditional growth medium supplemented with $10 \%$ FBS, revealed that the GBM neurospheres were tumorigenic, presented multilineage differentiation potential, and preserved the genotype of the original tumor, in contrast to the $10 \%$ FBS cultures which were not tumorigenic at low passages and considerably diverged from the original tumors at late passages ${ }^{4}$.

Isolation of CSC from dissociated GBM tumors by cell sorting based on the expression of putative CSC marker CD133 has also been proposed $^{6,7}$, but further work indicated that the CSC phenotype is not definitively associated with the expression of such markers ${ }^{8-10}$, decreasing the initial enthusiasm for this strategy, whereas new markers are still being tested ${ }^{10}$. The unavailability to date of a validated set of markers defining CSC, along with the goal of large scale amplification of these cells for preclinical studies, renders the use of cell sorting impractical for 
routine CSC-enriched cultures. GBM cells selected by the ability to grow as neurospheres in NMGF invariably express neural stem cell markers. We have observed that Sox2 and nestin are ubiquitously expressed in neurosphere cultures, while CD133 protein is present in a subset of the GBM neurospheres (unpublished data and reference ${ }^{11}$ ).

Several laboratories are pursuing neurosphere cultures from glioblastoma tumors using the same general approach of enzymatic dissociation and culturing in serum-free medium supplemented with growth factors $\mathrm{s}^{3,4,11-14}$, while other colleagues have reported attempts to grow long term neurosphere cultures from GBM samples without success. The general method for enzymatic dissociation and neurosphere culture of high grade gliomas presented here is similar to what has been outlined in the above publications. We have optimized the protocol based on our experience of dissociating and culturing over 100 GBM samples. The efficiency of obtaining long-term neurosphere cultures from fresh GBM samples applying the protocol presented here is over $40 \%$, similar to the few reports showing efficiency data, ${ }^{3,15}$, leading to the exploration of alternative protocols such as culturing cells continually or intermittently in serum-free medium in the presence of EGF and bFGF as monolayers on surfaces coated with ECM protein ${ }^{16,17}$. Neurosphere cultures are still the most validated and increasingly popular approach to preserve GBM tumors molecular characteristics and tumorigenic potential ${ }^{3,4,11-14}$, thus our approach is to attempt neurospheres cultures first, while concomitantly testing alternative methods for culturing GBM cells that fail to form long term self-renewing neurospheres (Figure 1), to increase the representation of GBM tumors that can be used in animal models. Here we present a protocol for culturing neurospheres from GBMs. For cells that fail to form neurospheres, we show a simple modification in the growth medium, as the first attempt to culture tumorigenic cells from nonneurosphere forming GBMs, with promising results and still undergoing extensive validation.

Protocol

\section{Single Cell Suspension from Fresh Surgical Glioblastoma Specimen for Neurosphere Culture}

1. With written consent from patients and in accordance with institutional guidelines, collect tumor samples for cell culture immediately after resection surgery of high grade glioma.

2. Confirm diagnosis of tumor sample by histopathology. Immediately transport the specimen from the operation room to the laminar flow tissue culture hood, for processing within $1 \mathrm{hr}$ from surgery.

NOTE: For surgeries at a remote site, cut the tumor sample into smaller fragments and place into a tube containing DMEM/F12 (keep on ice). The tumor can be processed several hours after surgery.

3. Starting with $200-500 \mathrm{mg}$ of tissue (optimal), mince the tumor sample using a scalpel blade, and transfer to a $15 \mathrm{ml}$ tube containing $10 \mathrm{ml}$ DMEM/F12 serum-free medium. Mix by inverting several times, let the tumor pieces sediment by gravity, remove medium, and repeat as necessary.

4. Prepare the Enzymatic Tissue Dissociation Solution and Stop Solution fresh.

1. Enzymatic Tissue Dissociation Solution: $5 \mathrm{ml}$ 0.05\% Trypsin-EDTA, $2.5 \mathrm{ml}$ Hank's Balanced Salt Solution (HBSS) calcium- and magnesium-free, $2.5 \mathrm{ml}$ Collagenase IV stock solution (2,000 U/ml in HBSS with calcium and magnesium).

2. Stop Solution: $5 \mathrm{ml}$ Trypsin Inhibitor solution, $5 \mathrm{ml}$ DMEM/F12, $2 \mu \mathrm{l}$ of 5,000 U/ml DNAse I (made in HBSS calcium- and magnesiumfree).

5. Remove medium and add Enzymatic Tissue Dissociation Solution to the minced tumor sample, using $2 \mathrm{ml}$ for each $0.5 \mathrm{~g}$ tissue. Gently mix by inverting.

6. Incubate the tissue in solution at $37^{\circ} \mathrm{C}$ in a tissue culture incubator under rotation for $30 \mathrm{~min}$.

7. Triturate with a $2 \mathrm{ml}$ pipette and stop the digestion or return to the incubator for another 15-30 min incubation depending on the level of digestion.

8. Stop digestion by adding 2 volumes of Stop Solution and triturate mechanically with a $5 \mathrm{ml}$ serological pipette.

9. Filter out the undigested material through a $40 \mu \mathrm{m}$ cell strainer. Pellet the cells at $800 \mathrm{xg}$ for $5 \mathrm{~min}$ at room temp, followed by washing $3 \times$ in $10 \mathrm{ml}$ DMEM/F12.

10. Resuspend the final cell pellet in $5 \mathrm{ml}$ DMEM/F12.

11. Slowly layer the cell suspension over $5 \mathrm{ml}$ of Lympholyte-M and pellet at $1,300 \mathrm{xg}$ for $20 \mathrm{~min}$ at room temperature.

12. Transfer the interface layer containing the nucleated cells to a $15 \mathrm{ml}$ tube containing $10 \mathrm{ml}$ of DMEM/F12.

13. Pellet the cells at $800 \times \mathrm{g}$ for $5 \mathrm{~min}$ at room temperature. Repeat the wash with DMEM/F12 $2 \times$ more.

14. Cryopreserve the resulting single cells for further use by resuspending the pellet in Recovery Cell Freezing Medium, aliquoting into cryovials, slow freezing, and storing in liquid nitrogen.

15. To culture, resuspend the cells in Neurosphere Medium supplemented with Growth Factors (NMGF), and plate at relatively low density ( $<1 \mathrm{x}$ $10^{5} \mathrm{cells} / \mathrm{ml}$ ) in a regular T25 tissue culture flask under standard conditions, $5 \% \mathrm{CO}_{2}, 37^{\circ} \mathrm{C}$, humidified tissue culture incubator (Figure $1 \mathrm{~A}$ ).

1. Prepare NMGF using the following recipe

\begin{tabular}{|l|l|l|}
\hline For $500 \mathrm{ml}$ of DMEM/F12 & & final concentration \\
\hline Stock solution & Volume & $1 \mathrm{x}$ \\
\hline N2 supplement (10x) & $5 \mathrm{ml}$ & $0.5 \mathrm{mg} / \mathrm{ml}$ \\
\hline $250 \mathrm{mg} / \mathrm{ml}$ BSA stock solution & $1 \mathrm{ml}$ & $25 \mu \mathrm{g} / \mathrm{ml}$ \\
\hline $10 \mathrm{mg} / \mathrm{ml}$ Gentamicin reagent & $1.25 \mathrm{ml}$ & $0.5 \mathrm{x}$ \\
\hline $100 \mathrm{x}$ antibiotic/antimycotic & $2.5 \mathrm{ml}$ & $20 \mathrm{ng} / \mathrm{ml}$ \\
\hline $100 \mathrm{mg} / \mathrm{ml} \mathrm{bFGF}$ & $0.1 \mathrm{ml}$ & \\
\hline
\end{tabular}




\begin{tabular}{|l|l|l}
$100 \mathrm{mg} / \mathrm{ml} \mathrm{EGF}$ & $0.1 \mathrm{ml}$ & $20 \mathrm{ng} / \mathrm{ml}$ \\
\hline
\end{tabular}

16. Transfer neurospheres that form over 1-3 weeks (Figures 1B-E) to fresh flasks, to separate from attached cells and debris. Perform partial media changes every 3-4 days.

NOTE: If dissociating orthotopic xenograft tumor using the protocol above, steps 1.11-1.13 can be omitted.

\section{Glioblastoma Neurosphere Culture Maintenance and Downstream Applications}

1. Dissociation: Monitor the neurosphere cultures and perform partial medium changes every 3-4 days. When most multicellular neurospheres in the flask reach $100 \mu \mathrm{m}$ in diameter, dissociate into a single cell suspension:

1. Transfer the medium containing the neurospheres to a $15 \mathrm{ml}$ tube, gravity sediment the neurospheres for about 5 min, remove the medium and resuspend the cell pellet in $10 \mathrm{ml}$ calcium- and magnesium-free DPBS.

2. Mix and incubate for $10 \mathrm{~min}$ at room temperature, as the cells sediment by gravity.

3. Remove $7-8 \mathrm{ml}$ of DPBS then dissociate the neurospheres mechanically with a prewetted serological pipette.

4. Pellet the dissociated cells at $800 \times \mathrm{g}$ for $5 \mathrm{~min}$ at $4{ }^{\circ} \mathrm{C}$.

2. Passaging: Resuspend the dissociated cells (step 2.1.) in NMGF and divide into the necessary number of flasks (1:3), and incubate under standard conditions. Secondary neurospheres will form, and should be subsequently dissociated for the formation of tertiary spheres.

3. Assessing long term self-renewal: Repeat the dissociation procedure until the neurosphere culture achieves at least 10 passages, equivalent to a minimum of 2 months in culture.

4. Freezing: To cryopreserve the neurospheres, dissociate the neurospheres (step 2.1.) and resuspend the cell pellet in Recovery Cell Freezing Medium, aliquot in cryovials, slow freeze, and store in liquid nitrogen.

1. To culture neurosphere cells from a frozen stock: thaw the cells at $37^{\circ} \mathrm{C}$ and immediately transfer to a $15 \mathrm{ml}$ tube containing $10 \mathrm{ml}$ $\mathrm{DMEM} / \mathrm{F} 12$. Mix the tube gently and then spin the cells at $800 \mathrm{xg}$ for $5 \mathrm{~min}$ at $4{ }^{\circ} \mathrm{C}$. Wash the cell pellet one more time and resuspend the cells in NMGF and transfer to a T25 tissue culture flask.

5. Cell suspension for intracranial implant in immunocompromised mouse:

1. Dissociate the neurospheres (step 2.1.) and resuspend the cell pellet in calcium- and magnesium-free DPBS. Count viable cells using a hemocytometer and trypan blue exclusion.

2. Calculate the number of cells needed for implant, typically $3 \times 10^{5}$ cells/mouse, transfer the desired number of cells into a vial, and pellet the cells at $1,000 \times \mathrm{g}$.

3. Resuspend the cell pellet in a volume appropriate for implant ( $5 \mu \mathrm{l} /$ mouse) by gently tapping the microcentrifuge tube. Place the cell suspension on ice and use within $2 \mathrm{hr}$.

\section{Alternative Protocol for Culturing Tumorigenic Glioblastoma Cells (for the cases where neurosphere culture fails)}

1. Supplement the NMGF medium with fetal bovine serum to a final concentration of $2 \% \mathrm{FBS} / \mathrm{NMGF}$

2. Starting with cells in NMGF culture: Harvest viable cells from the NMGF flask (Figure 1F), resuspend in $2 \%$ FBS/NMGF and plate at relatively low density $\left(<1 \times 10^{5} \mathrm{cells} / \mathrm{ml}\right)$ in a regular T25 tissue culture flask, and culture under standard conditions, $5 \% \mathrm{CO}_{2}, 37^{\circ} \mathrm{C}$, humidified tissue culture incubator (Figure 1G).

3. Starting with frozen never cultured cells (step 1.14.): Thaw the cells, wash in serum-free medium and plate in $2 \%$ FBS/NMGF and culture as described in step 3.2. Culturing in NMGF for 3 days prior to transferring viable cells to $2 \%$ FBS/NMGF is an alternative to preselect away differentiated cells.

\section{Morphological and Molecular Analysis of Neurospheres by Immunohistochemistry}

1. Culture the neurospheres until most floating multicellular spheroids reach $100 \mu \mathrm{m}$ diameter.

2. Remove culture flasks from the incubator and immediately pellet the neurospheres, remove medium and add $10 \mathrm{ml}$ of DPBS without disturbing the pellet. Remove DPBS, resuspend in 10\% neutral buffered formalin and incubate for 20 min at room temperature.

3. Pellet the spheroids, resuspend in $30 \%$ ethanol and incubate for $30-45 \mathrm{~min}$.

4. Replace $30 \%$ ethanol with $50 \%$ ethanol, incubate for $15 \mathrm{~min}$ and replace with fresh $50 \%$ ethanol, incubate a further $30 \mathrm{~min}$.

5. Repeat (step 4.4.) with $70 \%$ ethanol.

6. Replace with $95 \%$ ethanol two changes $10-20$ min each, and repeat with absolute ethanol until spheroids are bright white and condensed.

7. Make a small filter paper cone out of lens paper and place it into a small funnel in a beaker. Wet the lens paper with absolute alcohol.

8. Loosen the pellet slightly with fresh $100 \%$ ethanol, pour off excess ethanol and pour the spheroids through the lens paper cone, making sure they go to the tip of the cone. Rinse the tube with additional absolute ethanol and pour any remaining spheroids through the lens paper cone.

9. Remove the paper cone from the funnel and carefully move the spheroids into the bottom of the cone. Fold the paper into a square and make sure the spheroids are as securely enclosed as possible.

10. Transfer the packaged neurospheres to a cassette and transfer to an automatic tissue processor.

11. Program the automatic tissue processor as follows: absolute ethanol, $10 \mathrm{~min}$, xylene, $15 \mathrm{~min}(2 \mathrm{x})$, paraffin, $10 \mathrm{~min}(4 \mathrm{x})$.

12. Remove the cassette from the processor and place it on the heated part of a paraffin embedding system . Make sure the entire surface is free of fragments, dust or other debris and siphon out all paraffin from the forceps warming wells. Warm clean paraffin free forceps and add a small amount of paraffin to a warmed base mold.

13. Open the cassette, remove the packet and place it on the heated part of the embedding system. Carefully open the paper cone until the spheroids are visible. Gather as much of the material as possible with prewarmed forceps and transfer to the liquid paraffin in the base mold. 
14. Repeat as required to retrieve as many of the spheroids as possible. Gently scrape the tissue paper with a prewarmed clean razor blade that has been wiped with ethanol to collect any residual spheroids without getting any paper into the block.

15. Carefully break up any spheroid clumps with warm forceps. Move the spheroids away from the corners into a uniform central layer. Move the base mold to the cooling area to secure the spheroids in place, add the cassette, fill slowly with paraffin, and chill thoroughly.

16. The neurosphere paraffin block can be used for normal sectioning and immunohistochemistry.

\section{Representative Results}

We have applied the protocol described above to dissociate and culture 125 fresh surgical glioblastoma specimens (Figure 1), 88 newly diagnosed, and 37 recurrent tumors (Table 2), with approved patient consent and under institutional guidelines. The efficiency of the protocol for establishing long term neurosphere cultures was $41.6 \%$, and similar for newly diagnosed tumors and recurrent tumors (Table 2). For some GBM samples, neurospheres form in the first few days (Figures 1B and 1C), while for others longer culturing time is required (Figures 1D and 1E).

Efficiency of neurosphere formation was not exclusively dependent on the level of necrosis in the tissue, as exemplified by the results from a newly diagnosed tumor with high cell density (GBM1) and a recurrent and necrotic tumor (GBM2) processed according to Protocols 1 and 2, both yielding neurosphere cultures (Figure 2).

Testing the tumorigenic potential of each neurosphere culture in immunocompromised mice is the crucial validation of this approach for enrichment of CSCs. Using a protocol similar to previously described ${ }^{18}$, GBM1 and GBM2 neurospheres were implanted into the brains of immunocompromised mice, under institutional and IACUC animal care guidelines. The xenograft tumors present morphological characteristics of GBMs, such as invasion into the brain parenchyma and necrosis (Figure 2).

GBM3 was dissociated (Figure 1A) and cultured in neurosphere medium (Figures 1D and 1E), and in 2\% FBS/NMGF (Figures 1D, 1G, and 1H). Monolayer cells cultured in $2 \%$ FBS/NMGF and neurosphere cells cultured in NMGF were dissociated and the same number of cells were implanted in nude mouse, using the same procedure as in Figure 2. No differences in survival, tumor growth dynamics, or morphology were observed between the two preimplant culture methods (Figures 3A-C). Tumor growth characteristics do not change up to the latest passage tested, P20 for neurospheres, and P10 for 2\% FBS/NMGF. While neural stem cell markers, including Sox2, are downregulated in most primary GBM cells cultured in $10 \%$ FBS $^{3,4,11}$, NMGF supplemented with $2 \%$ FBS allows for the retention of Sox2 expression (Figure 3D).

Neurospheres were processed according to Protocol 4, and labeled with H\&E (Figure 4A), anti-Sox2 antibody, showing nuclear localization (Figure 4B), and EGFR antibody, cell membrane localization (Figure 4C). This protocol has been applied to access stimuli-dependent alterations in the expression of nestin, GFAP, and the proliferation marker Ki $67^{11}$.

Table 2. Efficiency of deriving long term self-renewing neurosphere cultures from GBMs.

\begin{tabular}{|l|l|l|}
\hline Pathology & $\mathbf{n}$ & $\begin{array}{l}\text { Percentage of samples yielding long term } \\
\text { self-renewing neurospheres }(\mathbf{n})\end{array}$ \\
\hline Glioblastoma - untreated, first surgery & 88 & $42.0 \%(37)$ \\
\hline Glioblastoma - recurrent & 37 & $40.5 \%(15)$ \\
\hline TOTAL & 125 & $41.6 \%(52)$ \\
\hline
\end{tabular}




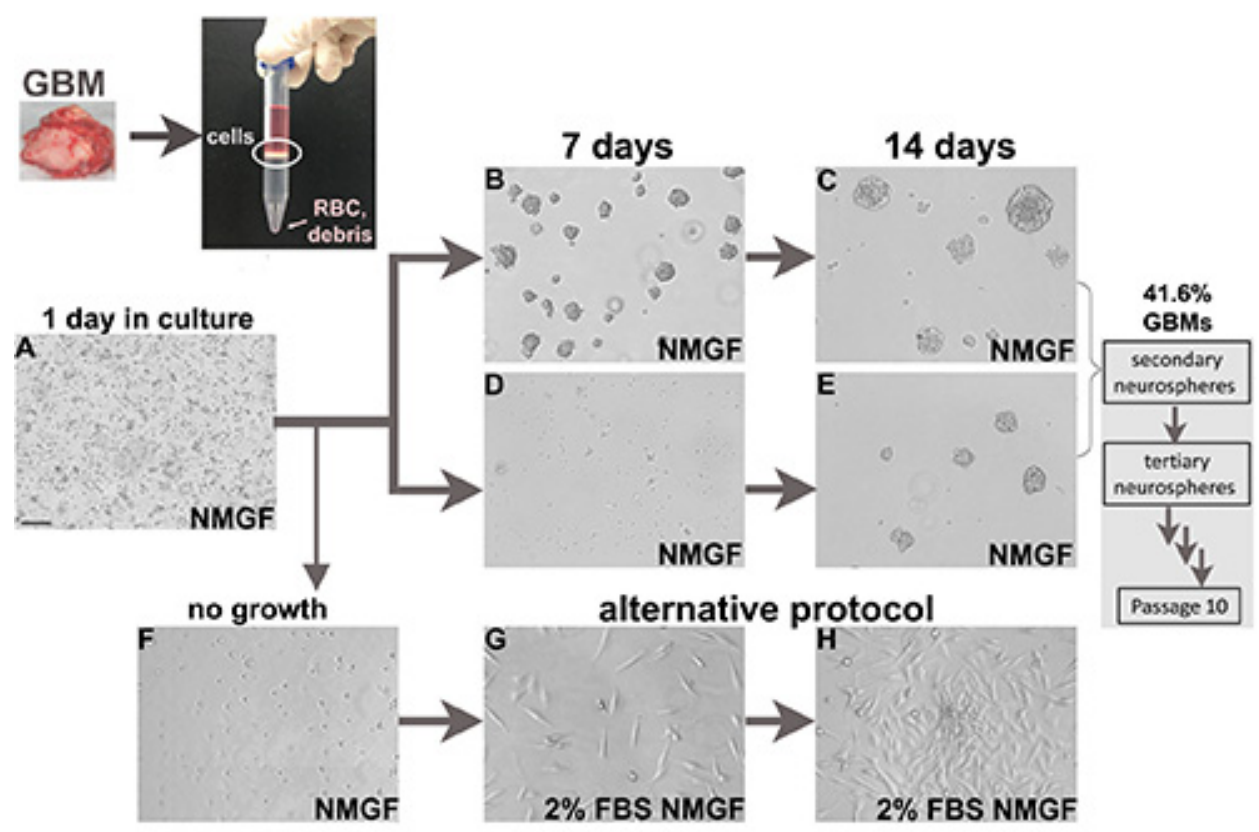

Figure 1. Cell culture from fresh glioblastoma surgical specimens. Fresh tumors are enzymatically dissociated into single cells. Nucleated cell interface from density separation medium is then plated in NMGF. Dissociated neurospheres are plated in NMGF, and typically 1 day after plating there are dead cells, debris, attached single cells, and occasional dividing cells in suspension (A). Neurosphere formation is faster for some cases (B,C), and slower for others (D,E). All neurospheres are dissociated and replated for at least 10 passages, $41.6 \%$ GBMs yield long term self-renewing neurospheres. Viable GBM dissociated cells that fail to grow as neurospheres $(\mathbf{F})$ can be transferred to alternative culture conditions, for example $2 \%$ FBS/NMGF (G,H). Bar (A), $100 \mu \mathrm{m}$, applies to all images. Click here to view larger image. 

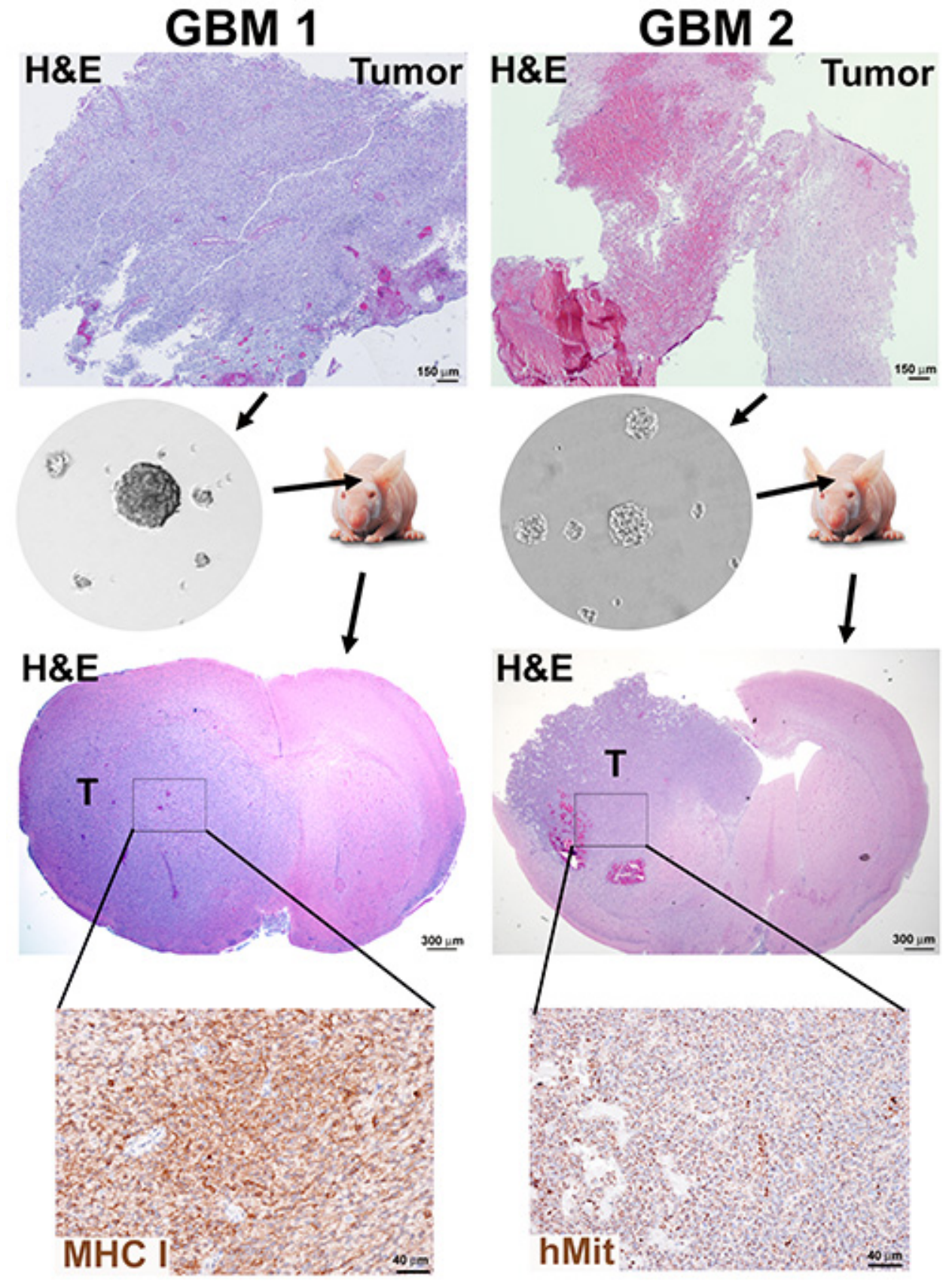

Figure 2. Neurosphere generation and orthotopic mouse xenografts from GBM tumors. A tumor sample presenting high cell density (GBM1) and a tumor with high necrotic content (GBM2) were dissociated and neurospheres cultured for 10 passages according to Protocols 1 and 2. Immunocompromised mice were implanted with $3 \times 10^{5}$ dissociated neurosphere cells and sacrificed when moribund. Mouse brains were formalin fixed and paraffin embedded for H\&E staining and immunohistochemistry detection of human markers, human mitochondria (hMit) or major histocompatibility class I subunit HLA-A (MHC-I). Click here to view larger image. 

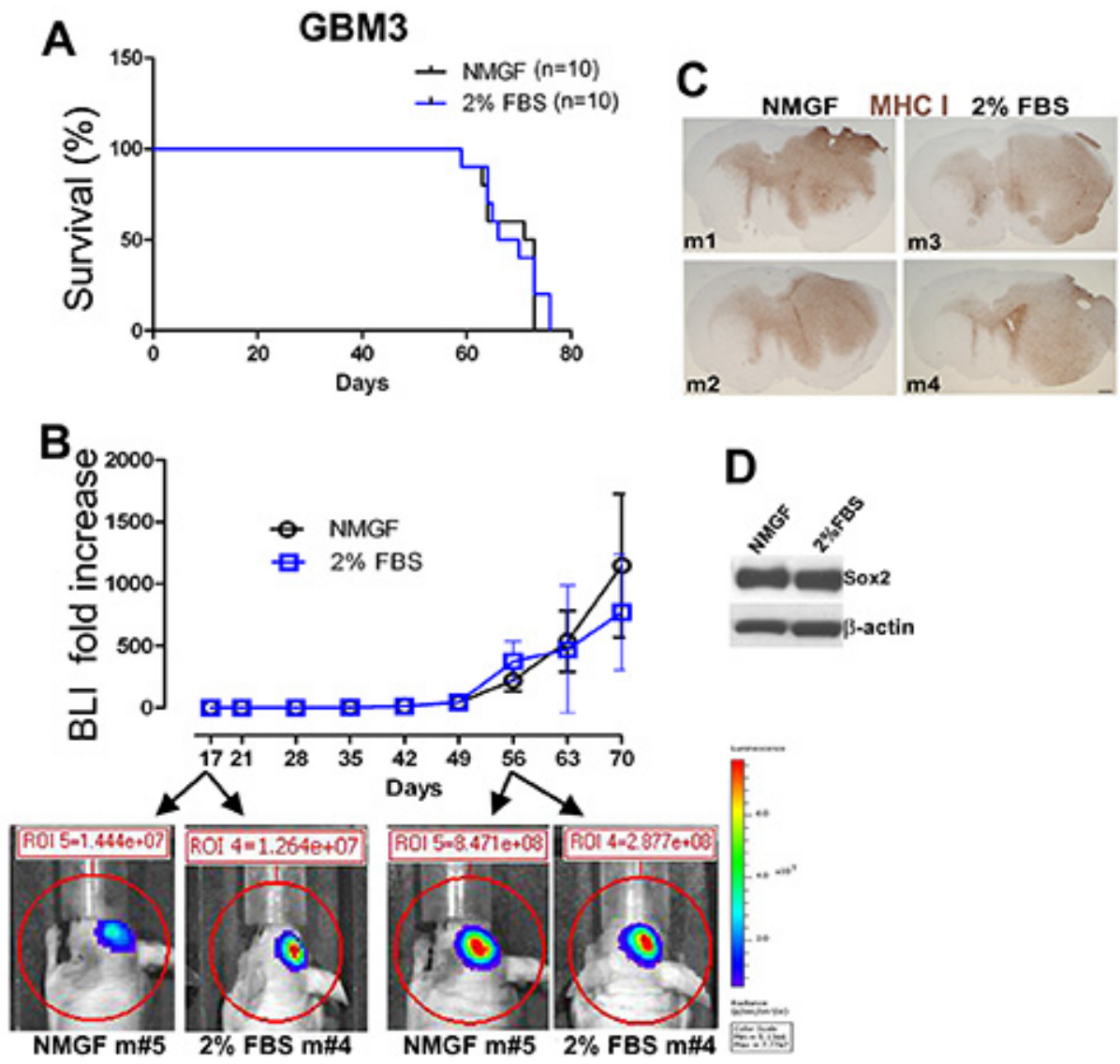

Figure 3. Similar tumor growth in mice implanted intracranially with GBM3 cells cultured as neurospheres in NMGF or as monolayers in $2 \%$ FBS/NMGF. (A) Kaplan-Meier survival curves for GBM3 cultured as either neurospheres in NMGF or monolayers in $2 \%$ FBS/NMGF (both passage $<10)$ show no difference in survival $(n=10, p=0.7174$, log-rank test). (B) Tumor growth monitored by live bioluminescence imaging (BLI) show no significant difference in tumor growth dynamics between the two groups. (C) Tumor morphology is indistinguishable for 4 GBM3 xenografts, 2 cultured in NMGF and 2 cultured in 2\% FBS/NMGF. MHC-I stain as described for Figure 2. Scale, $600 \mu \mathrm{m}$. (D) Western blot showing the stem cell maker Sox2 expression retained in 2\% FBS/NMGF cultures used to initiate the xenograft tumors (A-C). Click here to view larger image.
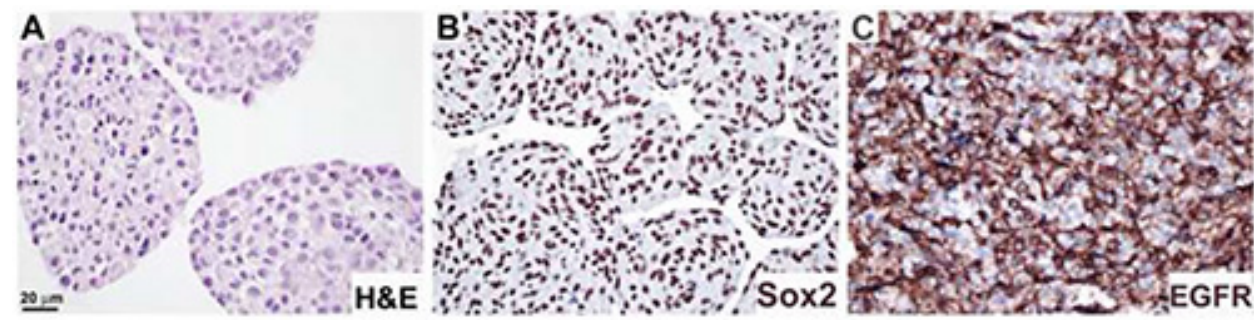

Figure 4. Analysis of protein expression in neurosphere sections by immunohistochemistry. Neurosphere cultures were formalin-fixed and paraffin embedded as described in Procedure 4, and stained with H\&E (A), anti-Sox2 antibody (B), and anti-EGFR antibody (C). DAB substrate was used to visualize (B,C). Bar, $20 \mu \mathrm{m}$. Click here to view larger image.

\section{Discussion}

Proper enzymatic tumor cell dissociation is a critical step in this protocol. The tissue must be minced well prior to incubation in cell dissociation solution at $37^{\circ} \mathrm{C}$ under rotation, for a minimum of $30 \mathrm{~min}$, when the tissue must be mechanically triturated and the extension of the dissociation verified. Depending on the degree of tissue cohesion, incubation can be extended for additional 15-30 min, as needed to complete dissociation into single cells. Incubation in dissociation solution for longer than $1 \mathrm{hr}$ is not recommended due to decreased cell viability. Although the optimal

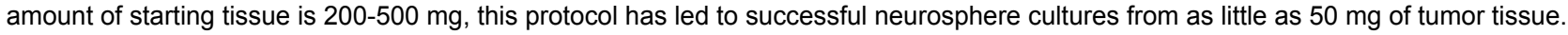

Serum-free NMGF is a selective medium, and a percentage of neoplastic cells comprising the bulk of the tumor, as well as host cells, will not survive, while others will attach to the tissue culture treated flask after plating. It is critical that the neurospheres that will form over 1-3 weeks (Figures 1B-E) are transferred to fresh flasks, to separate from differentiated tumor cells and debris. 
GBM cells enzymatically dissociated and never cultured (step 1.10) can be cryopreserved as a backup source of cells to culture in alternative media, in case the neurosphere culture fails. We have successfully obtained neurosphere cultures from such frozen stocks, as well as monolayer cells growing in $2 \%$ FBS/NMGF.

Conceptually, "cancer stem cell" is a work in progress and this emerging field will benefit from additional clarification, since the clinical implications are considerable, particularly in the generation of tumor heterogeneity, plasticity, and resistance to therapy ${ }^{19,20}$. In addition to being vital for generating patient-specific GBM animal models, the neurosphere cultures are also valuable for in vitro studies such as alteration in cell signaling and gene expression in response to growth factors, hypoxia, and pharmacological agents ${ }^{11,21}$. We have opted for using cross-sections of formalin fixed and paraffin embedded neurospheres, preserving the 3D architecture, to study protein expression and post-translational modifications by immunohistochemistry ${ }^{11}$, due to the superior subcellular localization in relation to the more common method of labeling and imaging the whole sphere.

It has been shown that not all cells within neurospheres derived from adult mammalian brain are stem cells ${ }^{22}$. Similarly, the neurospheres cultured from GBM tumors are likely not clonal, due to the presence of more differentiated cancer progenitor cells and self-aggregation, known to occur at high cell densities ${ }^{23}$, which is considered a limitation of this method by some. To favor enrichment for long term self-renewing stem cells, as opposed to just progenitors which can transiently grow as neurospheres ${ }^{22}$, the primary neurospheres are dissociated for secondary neurosphere formation, and further passaged for a minimum of 10 passages, roughly equivalent to 2 months in continuous culture, which is an indication of a population enriched in stem cells ${ }^{22}$. In our experience, GBM neurosphere cultures that achieve that mark can continue to be expanded as neurospheres indefinitely. Tumor grade matters, since we have obtained successful cultures from GBMs and anaplastic astrocytomas, WHO grade IV and III, respectively, but not from lower grade gliomas.

The most prevalent method of culturing glioblastoma cells, in traditional growth medium supplemented with $10 \%$ FBS, leads to considerable genomic and phenotypic divergence from the original tumors ${ }^{4}$. On the other hand, neurosphere cultures are a stable and long term source of cells presenting cancer stem cell phenotype ${ }^{4}$. The neurosphere method has become increasingly popular for the enrichment of CSC in long term cultures for orthotopic mouse xenograft models, despite not being successful for all high grade astrocytoma samples, which represents the main weakness of this method. Studies to understand how molecular characteristics of the parental tumors can affect neurosphere formation are underway. Exploration of practical alternative methods to culture high grade gliomas, followed by extensive validation are granted, given the momentous advantages of having patient specific GBM models, as we advance into an era of personalized medicine, fuelled by the accessibility of "omics" information and increased numbers of potential targeted therapies.

\section{Disclosures}

The authors declare that they have no competing financial interests.

\section{Acknowledgements}

This work has been funded by the Hermelin Brain Tumor Center, Henry Ford Hospital.

\section{References}

1. Singh, S. K. et al. Identification of a cancer stem cell in human brain tumors. Cancer Res. 63, 5821-5828 (2003).

2. Ignatova, T. N. et al. Human cortical glial tumors contain neural stem-like cells expressing astroglial and neuronal markers in vitro. Glia. 39 , 193-206 (2002).

3. Galli, R. et al. Isolation and characterization of tumorigenic, stem-like neural precursors from human glioblastoma. Cancer Res. 64, 7011-7021 (2004).

4. Lee, J. et al. Tumor stem cells derived from glioblastomas cultured in bFGF and EGF more closely mirror the phenotype and genotype of primary tumors than do serum-cultured cell lines. Cancer Cell. 9, 391-403 (2006).

5. Reynolds, B. A. \& Weiss, S. Generation of neurons and astrocytes from isolated cells of the adult mammalian central nervous system. Science. 255, 1707-1710 (1992).

6. Bao, S. et al. Glioma stem cells promote radioresistance by preferential activation of the DNA damage response. Nature. 444, 756-760, doi:10.1038/nature05236 (2006).

7. Singh, S. K. et al. Identification of human brain tumour initiating cells. Nature. 432, 396-401 (2004).

8. Beier, D. et al. CD133(+) and CD133(-) glioblastoma-derived cancer stem cells show differential growth characteristics and molecular profiles. Cancer Res. 67, 4010-4015 (2007).

9. Joo, K. M. et al. Clinical and biological implications of CD133-positive and CD133-negative cells in glioblastomas. Lab. Invest. 88, 808-815 (2008).

10. Son, M. J., Woolard, K., Nam, D. H., Lee, J. \& Fine, H. A. SSEA-1 is an enrichment marker for tumor-initiating cells in human glioblastoma. Cell Stem cell. 4, 440-452, doi:10.1016/j.stem.2009.03.003 (2009).

11. deCarvalho, A. C. et al. Gliosarcoma stem cells undergo glial and mesenchymal differentiation in vivo. Stem Cells. 28, 181-190, doi:10.1002/ stem.264 (2010).

12. Azari, H. et al. Isolation and expansion of human glioblastoma multiforme tumor cells using the neurosphere assay. J. Vis. Exp. e3633, doi:10.3791/3633 (2011).

13. Laks, D. R. et al. Neurosphere Formation Is an Independent Predictor of Clinical Outcome in Malignant Glioma. Stem Cells. 27, 980-987 (2009).

14. Vescovi, A. L., Galli, R. \& Reynolds, B. A. Brain tumour stem cells. Nat. Rev. Cancer. 6, 425-436 (2006).

15. Gunther, H. S. et al. Glioblastoma-derived stem cell-enriched cultures form distinct subgroups according to molecular and phenotypic criteria. Oncogene. 27 (20), 2897-2909 (2007). 
16. Fael Al-Mayhani, T. M. et al. An efficient method for derivation and propagation of glioblastoma cell lines that conserves the molecular profile of their original tumours. J. Neurosci. Methods. 176, 192-199, doi:10.1016/j.jneumeth.2008.07.022 (2009).

17. Pollard, S. M. et al. Glioma stem cell lines expanded in adherent culture have tumor-specific phenotypes and are suitable for chemical and genetic screens. Cell Stem cell. 4, 568-580, doi:10.1016/j.stem.2009.03.014 (2009).

18. Ozawa, T., James, C. D. Establishing Intracranial Brain Tumor Xenografts With Subsequent Analysis of Tumor Growth and Response to Therapy using Bioluminescence Imaging. J. Vis. Exp. (41), e1986, doi:10.3791/1986 (2010).

19. Leder, K., Holland, E. C. \& Michor, F. The therapeutic implications of plasticity of the cancer stem cell phenotype. PLoS One. 5, e14366, doi:10.1371/journal.pone.0014366 (2010).

20. Pietras, A. Cancer stem cells in tumor heterogeneity. Adv. Cancer Res. 112, 255-281, doi:10.1016/B978-0-12-387688-1.00009-0 (2011).

21. Lomonaco, S. L. et al. The induction of autophagy by gamma-radiation contributes to the radioresistance of glioma stem cells. Int. J. Cancer. 125, 717-722, doi:10.1002/ijc.24402 (2009).

22. Reynolds, B. A. \& Rietze, R. L. Neural stem cells and neurospheres--re-evaluating the relationship. Nat. Methods. 2, 333-336, doi:10.1038/ nmeth758 (2005).

23. Singec, I. et al. Defining the actual sensitivity and specificity of the neurosphere assay in stem cell biology. Nat. Methods. 3, 801-806, doi:10.1038/nmeth926 (2006). 